\title{
Area-metric analysis of the quality of obturation of four different techniques: An ex vivo study
}

\author{
Yazdan Shantiaee ${ }^{1}$, Setareh Rostami ${ }^{2}$, Shiva Shojaeeian ${ }^{3}$ and Sanaa Jabbari ${ }^{4}$ \\ ${ }^{1}$ Associate Professor, Dental School of Shahid Beheshti University of Medical Sciences, Tehran, Iran \\ ${ }^{2}$ Dentist, Shahid Beheshti University of Medical Scineces, Tehran, Iran \\ ${ }^{3}$ Assistant professor, Department of Endodontics, Dental school of Shahid Beheshti University of Medical \\ Sciences, Tehran, Iran \\ ${ }^{4}$ Post Graduate Student, Department of Endodontics, Dental School of Shahid Beheshti University of Medical \\ Sciences, Tehran, Iran
}

\begin{abstract}
The aim of this study was to compare different obturation techniques in straight canals in term of the percentage of gutta-percha filled area (PGFA) and percentage of sealer filled area (PSFA) and voids. The obturation time was also compared. Straight root canals of 40 extracted human premolars were instrumented with Mtwo rotary files and filled as follows: Control: cold lateral compaction with standard master and accessory cones. CLS group: cold lateral compaction with 0.04/35 master cone and standard accessory cones. CLNS group: cold lateral compaction with 0.04/35 master cone and non-standard medium-fine (MF) accessory cones. WLS group: warm lateral compaction with $0.04 / 35$ master cone and standard accessory cones. AH26 sealer was used. The teeth were sectioned horizontally at 3, 6 and $9 \mathrm{~mm}$ from the apex. The total area of each section and the PGFA, PSFA and percentage of voids were calculated. Data were analyzed using repeated measures ANOVA and Kruskal-Wallis test.The CLNS was the fastest method with a significant difference with the control group $(\mathrm{P}<0.05)$ while WLS required significantly more time than all other techniques $(\mathrm{P}<0.05)$. In the apical and coronal thirds, CLNS yielded significantly lower $\mathrm{PGFA}(\mathrm{P}<0.05)$. In the middle third, WLS yielded significantly higher PGFA than CLNS $(\mathrm{P}<0.05)$. Cold lateral compaction using gutta-percha master cone with 4\% taper and MF accessory cones is not efficient for obturation of canals. Also, warm lateral compaction has no superiority to cold lateral compaction in straight canals.
\end{abstract}

KEY WORDS: COLD LATERAL CONDENSATION, SEALER, WARM LATERAL COMPACTION, OBTURATION, GUTTA-PERCHA

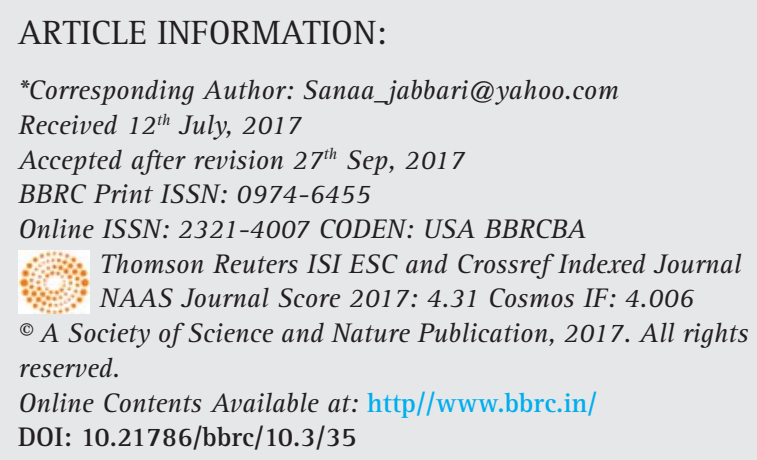




\section{INTRODUCTION}

Achieving a hermetic apical, lateral and coronal seal is an important factor to prevent entry of microorganisms into the canal and periapical tissues, and depends on proper cleaning and shaping and efficient obturation of root canal system (Wu et al. 1993; Mollander et al. 1998; Schafer et al. 2012). Advances in formulations of root canal irrigating solutions and rotary devices have greatly enhanced proper cleaning and shaping of root canal system. Homogenous, three-dimensional filling of root canal space by gutta-percha and sealer can greatly increase the success of endodontic treatment (Wu et al. 2002). Gutta-percha cones must be compacted next to each other with a very thin layer of sealer between gutta-percha cones and canal wall to minimize voids (Gordon et al. 2005). Gutta-percha dimensions do not change over time (Wu et al. 2009) but sealer undergoes shrinkage and dissolution (Al-Hadlaq et al. 2010; Nica et al. 2012). Following dissolution of sealer over time, voids are created in the canal, which can compromise the seal and enable entry and accumulation of microorganisms and subsequent development of periapical lesions and eventual treatment failure (Jarret et al. 2004; Schafer et al. 2012).

If the volume of gutta-percha mass compared to that of sealer increases, less voids will be created over time and coronal and apical seal will not be compromised. This would increase success of endodontic treatment (Kazemi et al. 1993; Kontakiotis et al. 1997; Wu et al. 2009). A systematic review reported that canals filled with adequate, homogenous gutta-percha mass showed primary endodontic success (Ng et al. 2008). Another study reported that homogeneity of gutta-percha mass had a significant positive effect on periapical health and success of endodontic treatment (Lee et al. 2012).

Root canal filling length should be equal to the working length to ensure that the entire prepared space is filled with gutta-percha (Kojima et al. 2004; Schaeffer et al. 2005). Thus, maximum penetration of master and accessory gutta-percha cones must be ensured. The taper of master and accessory gutta-percha cones is an important factor affecting the penetration depth of gutta-percha into the canal such that the greater the compatibility of taper of gutta-percha with that of root canal, the more homogenous and regular their arrangement would be in the canal. Some authors believe that using a single gutta-percha cone that matches the taper and size of prepared canal would have the best adaptation to canal walls (Whitworth, 2005; Schafer et al. 2013).

Several methods have been suggested for root canal obturation such as lateral compaction, vertical condensation, single-cone obturation with a gutta-percha cone that matches the size and taper of prepared canal
(Schafer et al. 2012), use of a solid core covered with gutta-percha, injection of soft, plasticized gutta-percha into the canal and use of physically and chemically modified gutta-percha with higher adaptation (Skinner et al. 1987; Whitworth, 2005; Schafer et al. 2013). Each of these techniques has its own advantages and disadvantages, and no consensus has been reached on the superiority of one technique over the others (Aqrabawi et al. 2006; $\mathrm{Ng}$ et al. 2007).

Lateral compaction is a commonly used root canal obturation method due to its simplicity (Cailleteau et al. 2009). This technique can be practiced in most clinical situations and enables length control during compaction (Gilhooly et al. 2001). However, its main drawback is that it cannot create a homogenous mono-block of gutta-percha. Master and accessory gutta-percha cones are placed next to each other but remain separate and the space between cones is expected to be filled with sealer, which undergoes shrinkage and dissolution over time. In thermoplastic methods such as warm vertical condensation and warm lateral compaction, heat is used to cause physical changes in gutta-percha and increase its plasticity and adaptation to the canal walls. Warm lateral compaction allows length control and at the same time, heat results in adherence of gutta-percha cones to each other and formation of a solid mass (Jacobsen et al. 1993).

Considering the need for decreasing working time in dental office, gutta-percha cones with 4\% and 6\% tapers and non-standard medium fine (MF) and fine-fine guttapercha cones were introduced to the market for faster obturation of root canals. However, deep penetration of accessory cones with greater taper is difficult. Studies on penetration depth of spreader alongside a master cone with $2 \%$ taper and master cones with higher than $2 \%$ taper showed greater penetration depth of spreader next to master cone with 2\% taper (Bal et al. 2001; Wilson et al. 2003). Thus, complete penetration of accessory cones to the working length and obtaining an apical seal with the use of these gutta-percha cones is a matter of debate. This study has aimed to assess the quality of root canal filling by measuring the percentage of gutta-percha (PGFA) and sealer filled areas (PSFA) and voids in use of four different obturation techniques. Duration of obturation was also compared among the four methods.

\section{MATERIAL AND METHODS}

This in vitro, experimental study was conducted on 40 single-canal maxillary and mandibular premolars with straight canals. The teeth were randomly divided into four groups $(n=10)$. The highest mean difference expected for PGFA among the groups was considered to 
be 0.13 with a standard deviation of $14 \%$ according to a study by Schafer et al. in 2012. Considering 80\% power of study and type one error of 5\%, sample size was calculated to be 10 in each group.

The inclusion criteria were single-rooted premolar teeth extracted for orthodontic or periodontal reasons with a straight canal and one apical foramen and no visible root caries, no fracture or crack and no internal/ external root resorption or calcification on radiographs. The teeth had mature apices.

The exclusion criteria were presence of voids after filling and incorrect working length. Teeth with no apical constriction or fracture during cleaning and shaping were also excluded. After collection, the teeth were immersed in 3\% sodium hypochlorite solution (Rakhshan, Tehran, Iran) at room temperature for two days. Calculus (if any) was removed using a universal curette (Hu-Friedy, USA) and the teeth were evaluated under a stereomicroscope (SZX9; Olympus, Tokyo, Japan) at 20 magnification to ensure absence of root cracks. Parallel periapical radiographs were obtained in mesiodistal and buccolingual dimensions to assess root canal anatomy. After access cavity preparation using 836 fissure and 801 round burs (SWS, Switzerland), a \#15 K file (Mani, Tokyo, Japan) was introduced into the canal until its tip was visible at the apex. The teeth were then radiographed and canal curvature was determined. The selected 40 teeth had straight canals and \#20 K file was the largest initial file compatible with their root canal. The crowns were then cut at the cementoenamel junction using a diamond disc (D \& Z Z, Germany).

To determine the working length, a \#15 K file (Mani, Japan) was introduced into the canal such that its tip was visible at the apex; working length was determined $1 \mathrm{~mm}$ short of this length. All canals were cleaned using \#15 and \#20 K files (Mani, Japan) and then by Mtwo rotary NiTi files (VDW, Germany) with an endodontic motor (NSK, Japan) with slow pull and push brushing motion to the working length as recommended by the manufacturer in the following order: 0.04/10, 0.05/15, 0.06/20, 0.06/25, 0.05/30, 0.04/35. A \# $10 \mathrm{~K}$ file (Mani, Japan) was used after each rotary file for patency and 17\% EDTA gel (Calasept, Nordiska Dental) was used as chelator. Root canals were rinsed with $2 \mathrm{cc}$ of $1.5 \%$ sodium hypochlorite (Rkhshan, Tehran, Iran). Smear layer was removed by rinsing the canals with $5 \mathrm{cc}$ of 17\% EDTA for one minute followed by a final rinse with saline. The root canals were dried with paper points (Ariadent, Tehran, Iran) and the teeth were randomly divided into four groups of 10 and filled with AH26 sealer (Dentsply DeTrey-Germany) and gutta-percha (Metabiomed, Korea) as follows:Control group: \#35 standard guttapercha ( $2 \%$ taper) as master cone and standard accessory cones were used in cold lateral compaction technique.
CLS groups: \#35 standard gutta-percha (4\% taper) as master cone and standard accessory cones were used in cold lateral compaction technique (cold lateral compaction with standard gutta-percha).CLNS group: \#35 standard gutta-percha (4\% taper) as master cone and non-standard MF accessory cones were used in cold lateral compaction technique (cold lateral compaction with non-standard gutta-percha).WLS group: \#35 standard gutta-percha (2\% taper) as master cone and standard accessory cones were used in warm lateral compaction technique (warm lateral compaction with standard guttapercha).Root canal filling with cold lateral compaction technique was done as follows: Following root canal preparation and drying, a standard gutta-percha cone with 2\% taper was used in the control group and with $4 \%$ taper in the CLS group to the working length. Apical third of master cone was dipped in sealer and placed into the canal, rotated in counter clockwise fashion and removed. A proper size spreader was also selected based on canal taper to reach $1 \mathrm{~mm}$ of the working length with master cone in the canal. Accessory cones were placed and cut at the orifice. No pressure was applied for vertical condensation.

Cold lateral compaction in CLNS group was the same as in the control and CLS groups with the exception of $4 \%$ taper of master cone and selected spreader. Also, nonstandard MF accessory cones were used. In WLS group, the same procedures were followed with the exception that after placement of master cone with $4 \%$ taper, the thinnest tip of Obtura (E \& Q Master Meta Biomed-Korea) that could reach $2-4 \mathrm{~mm}$ of the apex adjacent to master cone was chosen and inserted as deep as possible into the canal. After removal, \#25 finger spreader was placed adjacent to the heated master cone, removed immediately and replaced with an accessory cone with $2 \%$ taper. This process was repeated until complete obturation. Time required for obturation of each canal was also calculated from the time of placement of the first gutta-percha to completion of obturation using a chronometer (reported in minutes). A control radiograph was also obtained from the mesiodistal direction to ensure complete obturation.

The teeth were incubated at $37^{\circ} \mathrm{C}$ and $100 \%$ moisture for 14 days for completion of setting of sealer. Using an endodontic motor, root surface was marked at 3, 6 and $9 \mathrm{~mm}$ from the apex and the teeth were fixed to IsoMet cutting machine (Buehler IsoMet Low Speed Saw 11-1180) from the coronal part using glue. The roots were sectioned horizontally under copious water. The slices were immersed in sodium hypochlorite solution for one minute to eliminate dentin chips and were then rinsed with 17\% EDTA (Calasept Nordiska Dental) for one minute followed by a rinse with water. The slices were photographed at $\times 40$ magnification under a stereomicroscope (SZX9; Olympus, Japan). 
Photographs were saved in TIF format and assessed in Adobe Photoshop CS6 1301.2 software. In this software, the total surface area of the canal in each section and the percentage of gutta-percha filled area (PGFA) and percentage of sealer-filled area (PSFA) were separately calculated on each image in pixels. The percentage of void areas [total area of section-(PSFA+PGFA)] was also calculated. These values were reported for each section. All measurements were made by an examiner blinded to the group allocation of teeth (Figure 1). The values were recorded in Microsoft Excel 2013.

The PGFA and PSFA data in different sections were analyzed using repeated measures ANOVA. Obturation time data were analyzed using ANOVA and data of percentage of voids were analyzed using the Kruskal Wallis test. The mean and standard deviation of PGFA, PSFA and void area were reported. For the comparison of the mean PGFA and PSFA, repeated measures ANOVA was used (since data were normally distributed). The Kruskal Wallis test was used to compare the mean area of voids in different sections. Data were analyzed using SPSS version 21 (SPSS Inc., IL, USA).

\section{RESULTS AND DISCUSSION}

Table 1 shows the mean PSFA, PGFA and void area values in apical, middle and coronal sections. Table 2 shows the mean time of obturation in the groups. Table 3 shows the $\mathrm{P}$ values for the comparisons. As seen in Table 3, in the apical section, CLNS group showed significantly lower PGFA than other groups $(\mathrm{P}<0.05)$. PGFA of WLS group was significantly higher than that of other groups followed by CLS, control and CLNS groups, respectively. The difference of WLS with CLNS was significant $(\mathrm{P}<0.05)$ but its difference with other groups was not significant $(\mathrm{P}>0.05)$ in this respect. The CLS group showed higher PGFA than the control group but this difference was not significant $(\mathrm{P}>0.05)$.

In the middle section, WLS showed higher PGFA than other groups followed by CLS, control and CLNS groups, respectively but only the difference between WLS and CLNS was significant $(\mathrm{P}<0.05)$. The CLNS group showed lower PGFA than other groups but this difference only for WLS was significant $(\mathrm{P}<0.05)$. The CLS showed higher PGFA than the control group but this difference was not significant $(\mathrm{P}>0.05)$.In the coronal section, the CLNS group showed significantly lower PGFA than other groups $(\mathrm{P}<0.0001)$. The PGFA in WLS group was higher than that in other groups followed by CLS, control and CLNS groups. The difference between CLNS and WLS was significant $(\mathrm{P}<0.0001)$ but other differences were not significant $(\mathrm{P}>0.05)$. The CLS group showed higher PGFA than the control group but this difference was not significant $(\mathrm{P}>0.05)$.
As seen in Table 4, in the apical section, the CLNS group showed significantly higher PSFA than other groups $(\mathrm{P}<0.05)$. The PSFA in WLS group was lower than that in other groups followed by CLS, control and CLNS groups, respectively. The difference between WLS and CLNS was significant $(\mathrm{P}<0.0001)$ but other differences were not statistically significant $(\mathrm{P}>0.05)$. The CLS group showed lower PSFA than the control group but this difference was not significant $(\mathrm{P}>0.05)$. In the middle section, WLS group showed lower PSFA than other groups followed by CLS, control and CLNS groups, respectively. Only the difference between the WLS and CLNS groups was significant $(\mathrm{P}<0.05)$. The CLNS group showed higher PSFA than other groups but this difference only with WLS was significant $(\mathrm{P}<0.05)$. The CLS group showed lower PSFA than the control group but this difference was not significant $(\mathrm{P}>0.05)$.

In the coronal section, the CLNS group showed significantly higher PSFA than other groups $(\mathrm{P}<0.0001)$. PSFA of WLS group was lower than that of other groups followed by CLS, control and CLNS groups, respectively. The difference of WLS and CLNS was significant $(\mathrm{P}<0.0001)$ but the difference with other groups was not statistically significant $(P>0.05)$. In general, in all sections, the WLS group showed higher PGFA and lower PSFA than other groups followed by CLS, control and CLNS groups, respectively. The difference between WLS and CLNS in terms of PGFA and PSFA only in the middle third was statistically significant $(\mathrm{P}<0.05)$. The CLNS group in all sections showed lower PGFA and higher PSFA than other groups and this difference in the apical and coronal section was statistically significant. The CLS group compared to the control group showed higher PGFA and lower PSFA in all sections but these differences were not statistically significant $(\mathrm{P}>0.05)$.

No significant difference was noted in apical, middle and coronal sections of the four groups in terms of voids $(\mathrm{P}>0.05)$.

As seen in Table 5, the mean time required for root canal obturation in WLS group was significantly longer than that in other groups $(\mathrm{P}<0.0001)$ followed by the control, CLS and CLNS groups, respectively. The mean time required for root canal obturation in CLNS group was significantly shorter than that in the control group $(\mathrm{P}<0.05)$.

Three-dimensional obturation of root canal system is necessary to prevent bacterial reentry and ensure longterm success of endodontic treatment (Raina et al. 2007; Michaud et al. 2008). Finding an efficient technique with the greatest ability to provide a hermetic seal is important to guarantee the success of endodontic treatment (Haikei et al. 2006). This study compared the quality of obturation of straight root canals prepared with Mtwo rotary files and filled with four obturation techniques by measuring the surface area filled with gutta-percha and 
Yazdan Shantiaee et al.

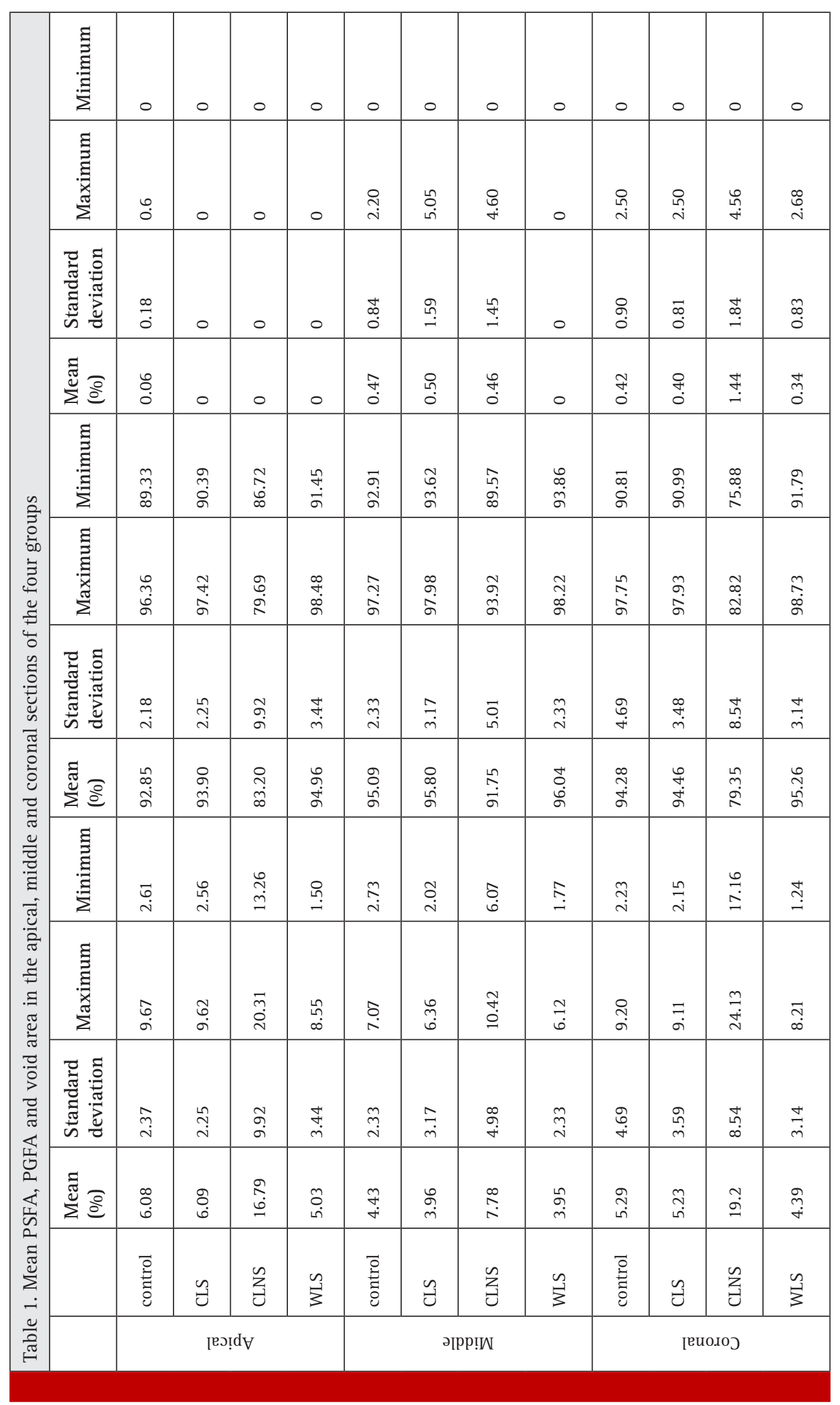




\begin{tabular}{|l|c|c|c|c|}
\hline \multicolumn{6}{|c|}{ Table 2. Obturation time in the four groups } \\
\hline Group & $\begin{array}{c}\text { Mean } \\
\text { (minutes) }\end{array}$ & $\begin{array}{c}\text { Standard } \\
\text { deviation }\end{array}$ & Maximum & Minimum \\
\hline Control & 6.41 & 0.60 & 7.60 & 5.20 \\
\hline CLS & 5.66 & 0.82 & 6.90 & 4.50 \\
\hline CLNS & 5.03 & 0.82 & 6.20 & 3.90 \\
\hline WLS & 8.43 & 1.18 & 10.30 & 6.50 \\
\hline
\end{tabular}

that root canals filled with gutta-percha of Mtwo rotary system had higher quality of obturation compared to those filled with gutta-percha of Reciproc and WaveOne systems. Also, we wanted to compare the percentage of area filled with gutta-percha in case of using guttapercha with 4\% taper. Thus, Mtwo was a suitable choice for this purpose since \#35 gutta-percha in Mtwo system has $4 \%$ taper. Although master cone in our study was \#35 with 4\% taper (matched single cone of Mtwo system), not all the prepared canals follow the shape and

\begin{tabular}{|c|c|c|c|c|c|c|c|c|c|c|c|c|}
\hline & \multicolumn{3}{|c|}{ WLS } & \multicolumn{3}{|c|}{ CLNS } & \multicolumn{3}{|c|}{ CLS } & \multicolumn{3}{|c|}{ Control } \\
\hline & Coronal & Middle & apical & coronal & Middle & apical & coronal & middle & apical & Coronal & Middle & Apical \\
\hline Control & 1 & 1 & 1 & $<0.0001^{*}$ & 0.203 & $0.001^{*}$ & 1 & 1 & 1 & & & \\
\hline CLS & 1 & 1 & 1 & $<0.0001^{*}$ & 0.067 & $0.001^{*}$ & & & & 1 & 1 & 1 \\
\hline CLNS & $<0.0001^{*}$ & $0.045^{*}$ & $<0.0001^{*}$ & & & & $<0.0001^{*}$ & 0.067 & $0.001^{*}$ & $<0.0001^{*}$ & 0.203 & $0.001^{*}$ \\
\hline WLS & & & & $<0.0001^{*}$ & $0.045^{*}$ & $<0.0001^{*}$ & 1 & 1 & 1 & 1 & 1 & 1 \\
\hline
\end{tabular}

\begin{tabular}{|c|c|c|c|c|c|c|c|c|c|c|c|c|}
\hline & \multicolumn{3}{|c|}{ WLS } & \multicolumn{3}{|c|}{ CLNS } & \multicolumn{3}{|c|}{ CLS } & \multicolumn{3}{|c|}{ Control } \\
\hline & Coronal & middle & apical & coronal & Middle & apical & coronal & middle & apical & Coronal & Middle & Apical \\
\hline control & 1 & 1 & 1 & $<0.0001^{*}$ & 0.206 & $0.002^{*}$ & 1 & 1 & 1 & & & \\
\hline CLS & 1 & 1 & 1 & $<0.0001^{*}$ & 0.068 & $0.001^{*}$ & & & & 1 & 1 & 1 \\
\hline CLNS & $<0.0001^{*}$ & $0.046^{*}$ & $<0.0001^{*}$ & & & & $<0.0001^{*}$ & 0.068 & $0.001^{*}$ & $<0.0001^{*}$ & 0.206 & $0.002^{*}$ \\
\hline WLS & & & & $<0.0001^{*}$ & $0.046^{*}$ & $<0.0001^{*}$ & 1 & 1 & 1 & 1 & 1 & 1 \\
\hline
\end{tabular}

sealer using area-metric analysis. The results showed that root canal obturation with non-standard accessory cones and a master cone with 4\% taper decreased the gutta-percha mass and increased the sealer area. Use of a master cone with 4\% taper had no positive effect on quality of obturation or working time. Warm lateral compaction in straight canals had no superiority over cold lateral compaction and took longer to accomplish.

In our study, root canals were prepared using Mtwo rotary system since Schafer et al. (2016) in 2016 showed

Table 5. P values for the comparison of obturation
time in the four groups
\begin{tabular}{|l|c|c|c|c|}
\hline Group & WLS & CLNS & CLS & Control \\
\hline Control & $<0.0001$ & 0.01 & 0.28 & - \\
\hline CLS & $<0.0001$ & 0.43 & - & 0.28 \\
\hline CLNS & $<0.0001$ & - & 0.43 & 0.01 \\
\hline WLS & - & $<0.0001$ & $<0.0001$ & $<0.0001$ \\
\hline
\end{tabular}

taper of final file after preparation with the final rotary file, so we designed a group in which we placed accessory gutta-percha cones between single cone and canal walls to assess the possibility of improving the quality of obturation. Apical seal is an important parameter in success of endodontic treatment and improper apical seal results in development of periapical lesions. The results of our study showed that in the apical third, use of non-standard gutta-percha alongside the master cone with 4\% taper significantly decreased the apical seal and quality of obturation and decreased PGFA and increased PSFA. Also, our results showed that use of standard gutta-percha with $2 \%$ and $4 \%$ taper along with $2 \%$ accessory cones did not cause a significant change in quality of obturation in the apical third. Although PGFA in 4\% gutta-percha group was higher and PSFA was lower than that in other groups, these differences were not significant and no difference in voids was noted either. Based on our results, warm lateral com- 
paction had no superiority over cold lateral compaction since no difference was noted between the two groups in any of the assessed parameters.

Comparison of the mean time of obturation among the groups showed that the mean time of obturation was significantly longer in WLS group compared to other groups. This time in CLNS group was significantly shorter than that in the control group. The CLS and control groups were not significantly different in this regard. Schafer et al. in 2012 compared the quality of root canal obturation with $2 \%$ and $4 \%$ taper gutta-percha cones by microscopic area-metric analysis and found no significant difference between the two types in the apical region. None of them provided a better apical seal. Romina et al.(2009) performed area-metric analysis of root filling and showed equal apical seal in use of gutta-percha cones with $2 \%$ and 4\% taper. Our results were in agreement with those of the afore-mentioned studies.

We found no significant difference in time of obturation between the use of gutta-percha cones with $2 \%$ and 4\% taper, which was in agreement with the results of Schafer et al.(2012). Our results regarding the use of non-standard MF gutta-percha cones in comparison with the use of standard gutta-percha showed that use of non-standard cones along with a master cone with 4\% taper decreased PGFA and increased PSFA in all sections and this difference in the apical and coronal thirds was significant. Thus, use of MF accessory cones along with 4\% gutta-percha does not provide suitable apical seal. In contrast to our results, VanGheluwe and Wilcox in 1996 found no significant difference in apical seal of standard and non-standard gutta-percha cones. Difference in the results of the two studies may be due to the use of curved roots in their study. Use of warm lateral compaction method in our study increased PGFA and decreased PSFA in all sections, although insignificantly. No difference was noted in terms of voids. No significant difference between warm and cold lateral compaction techniques in our study may be due to our small sample size and use of straight canals because cold lateral compaction in straight canals is much easier and yields better results than in curved canals.

Thus, future studies with larger sample sizes are required to compare these two methods in curved canals. Collins et al. (2006) compared warm and cold lateral compaction and warm vertical condensation and concluded that warm lateral compaction significantly increases the volume of gutta-percha mass in the canal compared to cold lateral compaction. Difference between our results and theirs may be due to different methodology since we performed area-metric analysis while they used replication of canal irregularity. Moreover, they used D11T spreader in their study, which is very fine and made of stainless steel, and can penetrate to $1 \mathrm{~mm}$ of the working length while we used finger spreader penetrated to $2 \mathrm{~mm}$ of the working length. Moreover, they used non-standard MF gutta-percha while we used standard cones with $2 \%$ taper.

Several methods are available for assessment of the quality of root canal filling such as dye penetration (McRobert et al. 997), microbial leakage models, replication of canal irregularity (Jacobson et al. 2008) and three-dimensional analysis by micro-computed tomography (CT) (Hammad et al. 2009). These methods have advantages and limitations. Dye penetration and microbial leakage are more commonly used due to simplicity and acceptable accuracy; however, smaller size of dye particles than some bacteria (Nielsen et al. 1995) and complexity and time consuming nature of microbial leakage (Sequeira et al. 2000) are among the drawbacks of these techniques.

Area-metric analysis has been used by many researchers for assessment of the success of endodontic treatment in vitro. Thus, this method was used in our study. The limitations of this method include two-dimensional analysis of the root canal filling and lower accuracy than micro-CT. Moreover, dentin chips and smear layer created during sectioning in this method may decrease the quality of images and cause erroneous determination of borders between gutta-percha, sealer and voids. To overcome this limitation, we immersed the slices in sodium hypochlorite solution for one minute after sectioning followed by one minute in 17\% EDTA and then rinsed them to eliminate the smear layer and dentin chips. Considering the higher accuracy of micro-CT for three-dimensional assessment of quality of obturation, further studies with the use of micro-CT on both straight and curved canals are required to obtain more accurate information on this topic.

\section{CONCLUSION}

Root canal obturation with non-standard accessory cones and a master cone with 4\% taper decreases the gutta-percha mass and increases the sealer area and therefore, compromises the quality of root canal filling. Use of a master cone with $4 \%$ taper has no positive effect on quality of obturation or working time. Warm lateral compaction in straight canals has no superiority over cold lateral compaction and takes longer to accomplish.

\section{REFERENCES}

Al-Hadlaq SM, Al-Jamhan A, Alsaeed T. (2010) Comparison of the single cone and lateral compaction techniques in sealing 0.04 taper root canal preparation. Gen Dent 58:219-22.

Aqrabawi JA. (2006) Outcome of endodontic treatment of teeth filled using lateral condensation versus vertical compaction. J Contempt Dent Pract 7:17. 
Bal AS, Hicks ML, Barnett F. (2001) Comparison of laterally condensed 0.06 and 0.02 tapered gutta-percha and sealer in vitro. J Endod 27:786-8.

Cailleteau JG, Mullaney TP. (1997) Prevalence of teaching apical patency and various instrumentation and obturation techniques in united states dental schools. J Endod 23:394.

Collins J, Walker MP, Kulild J, Lee C.A comparison of three gutta-percha obturation techniques to replicate canal irregularities. J Endod 2006;8:762-5.

Gilhooly RM, Hayes SJ, Bryant ST, Dummer PM. (2001) Compacted of lateral condensation and thermomechanically compacted warm alpha-phase gutta-percha with a single cone for obturating curved root canals. Oral Surg Oral Med Oral Pathol Oral Radiol Oral Endod 91:89.

Gordon MPJ, Love R, Chandler NP. (2005) An evaluation of 0.06 tapered gutta-percha cones for filling of 0.06 taper prepared curved root canals. Int Endod J38:87-96.

Haikei Y, Freymann M, Fanti V, Claisse A, Poumier F, Watson M. (2006) Apical microleakage of radiolabeled lysozyme over time in three techniques of root canal obturation. J Endod 26:148-52.

Hammad M, Qualtrough A, Silikas N. (2009) Evaluation of root canal obturation: a three dimensional in vitro study. J Endod 35:541-4.

Jacobsen EL, Begole EA.( 1992) A Comparison of four canal obturation methods employing gutta-percha: a computerized analysis of the internal structure. Endod Dent Traumatol 8:206.

Jacobson HLJ, Xia T, Mini-Kai W, Paul RW. (2008) Sealing ability of a new polydimethylsiloxane-based root canal filling materials. J Endod 34:204-7.

Jarret IS, Marx D, Covey D, Karmazin M, Lavin M, Gound T. (2004) Percentage of canals filled in apical cross sections. Int Endod J 37:392-8.

Kazemi RB, Safavi KE, Spangberg LSW. (1993) Dimensional changes of endodontic sealers. Oral Surg Oral Med Oral Pathol 76:766-71.

Kojima K, Inamoto K, Nagamatsu K. (2004) Success rate of endodontic treatment of teeth with vital and non-vital pulps: a meta-analysis. Oral Surg Oral Med Oral Pathol Oral Radiol Oral Endod 97:5-9.

Kontakiotis EG, Wu MK, Wesselink PR.( 1997) Effect of sealer thickness on long-term sealing ability: a 2-year following study. Int Endod J 30:307-12.

Lee AH, Cheung GS, Wong MC. (2012) Long term outcome of primery non-surgical root canal treatment. Clin Oral Invest 16:1607-17.

McRobert AS, Lumley PJ. An in vitro investigation of coronal leakage with three gutta-percha back filling techniques. Int Endod J 1997;30:413-7.

Michaud R, Burgess J, Barfield R, Cakir D, McNeal S, Eleazer P. (2008) Volumetric expansion of gutta-percha in contact with eugenol. J Endod 34:1528-32.
Mollander A, Reit C, Dahlen G, Kvist T. (1998) Microbiological status of root-filled teeth with apical periodontitis. Int Endod $\mathrm{J} 31: 1-7$

Ng YL, Mann V, Rahbaran S. (2008) Outcome of primery root canal treatment: systematic review of the literature. Int Endod J 41:6-31.

Ng YL, Mnn V, Rahbaran S, Lewsey J, Gulabivala K. (2007) Outcome of primery root canal treatment: systematic review of the literature. Int Endod J 40:921.

Nica LM, Didilescu A, Rusu D, Bacila A, Stratul SI. (2012) Photomicrographic evaluation of the apical sealing capacity of three types of gutta-percha master cones: an in vitro study. Odontology 100:54-60.

Nielsen RB, Alyassin AM, Peters DD. (1995) Micro computed tomography: an advanced system for detailed endodontic research. J Endod 21:561.

Raina R, Loushine RJ, Weller RN, Tay FR, Pashley DH. (2007) Evaluation of the quality of the apical seal in Resilon/Epiphany and gutta-percha/AH plus-filled root canal by using fluid filtration approach. J Endod 33:944-7.

Romina C, Beltes P, Boutsionkis C, Dandakis C. (2009) Ex-vivo area-metric analysis of root canal obturation using gutta-percha cones of different tapers. Int Endod J 42:491-8.

Schaeffer MA, White RR, Walton RE. (2005) Determining the optimal obturation length: a meta-analysis of literature. J Endod 31:271-4.

Schafer E, Koster M, Burklein S. (2013) Percentage of guttapercha filled areas in canals instrumented with NiTi systems and obturated with matching single cones. J Endod 39: 924-4.

Schafer E, Neilus B, Burklein S. (2012P) A comparative evaluation of gutta-percha filled area in curved root canals obturated with different techniques. Clin Oral Invest 16:225-30.

Schafer E, Schrenker C, Zupanc J, Burklein S. (2016) Percentage of gutta-percha filledarea in canals obturated with crosslinked gutta-percha core-carrier systems, single-cone and lateral compaction technique. J Endod 2:294-8.

Sequeira JF, Rocas IN, Favieri A, Amad EC, Castro AJR, Gahyva SM. (2000) Bacterial leakage in coronaly unsealed root canals obturated with 3 different techniques. Oral Surg Oral Med Oral Pathol 90:647-50.

Skinner RL, Himel VT. (1987) The sealing ability of injectionmodeled thermoplasticized gutta-percha with and without the use of sealers. J Endod 13:315.

VanGheluwe J, Wilcox LR. (1996) Lateral condensation of small curved root canals root canals: comparison of two types of accessory cones. J Endod 22:540-42.

Whitworth J. Methods of filling root canals: principles and practices. Endod Topics. 2005;12:2-24.

Wilson J, Baumgartner JC. (2003) Comparison of spreader penetration during lateral compaction of 0.04 and 0.02 guttapercha. J Endod 29:828-31. 
Wu MK, Bud MG, Wesselink PR. (2009) The quality of single cone and laterally compacted gutta-percha fillings in small and curved root canals as evidenced by bidirectional radiographs and fluid transport measurements. Oral Surg Oral Med Oral Pathol Oral Radiol Endod 108:946-51.
Wu MK, Kast Akora A, Wesselink PR. (2002) Quality of cold and warm gutta-percha fillings in oval canals in mandibular premolars. Int Endod J34: 485-91.

Wu MK, Wesselink PR. (1993) Endodontic leakage studies reconsidered methodology, application and relevance. Int Endod J26:37. 